\title{
Differences in Complexion between Cold- and Heat-Prescription Groups in Sasang Medicine
}

\author{
Young Joo Park, ${ }^{1,2}$ Jun-Hyeong Do, ${ }^{1,2}$ Honggie Kim, ${ }^{3}$ and Jong Yeol Kim ${ }^{1,2}$ \\ ${ }^{1}$ KM Fundamental Research Division, Korea Institute of Oriental Medicine (KIOM), 1672 Yuseong-daero, \\ Yuseong-gu, Daejeon, Republic of Korea \\ ${ }^{2}$ Korean Medicine Life Science, University of Science and Technology, 217 Gajeong-ro, Yuseong-gu, Daejeon, Republic of Korea \\ ${ }^{3}$ Department of Information and Statistics, Chungnam National University, 99 Daehak-ro, Yuseong-gu, Daejeon, Republic of Korea
}

Correspondence should be addressed to Honggie Kim; honggiekim@cnu.ac.kr and Jong Yeol Kim; ssmed@kiom.re.kr

Received 17 March 2017; Revised 7 July 2017; Accepted 10 July 2017; Published 14 August 2017

Academic Editor: Ki-Wan Oh

Copyright (C) 2017 Young Joo Park et al. This is an open access article distributed under the Creative Commons Attribution License, which permits unrestricted use, distribution, and reproduction in any medium, provided the original work is properly cited.

\begin{abstract}
Sasang constitutional medicine (SCM) is a type of traditional Korean medicine (TKM) that classifies the human constitution into four types. The appearance of the complexion is one of the diagnostic factors of SCM but is rarely used in a quantitative and objective manner for diagnosis. In this study, an analysis using actual clinical data was conducted to assess the use of the complexion as a diagnostic element. A total of 528 Sasang medicine prescriptions from Korea Constitutional Multicenter Bank (KCMB) were classified into either a Cold-prescription group or a Heat-prescription group, and the complexion differences of the patients were analyzed using the $L^{*} a^{*} b^{*}$ color space. After adjusting for age, BMI, and systolic blood pressure, significant differences were observed among the Cold- and Heat-prescription groups within each Sasang constitution. However, when the Sasang constitution was ignored, no significant difference was observed for either sex. This study quantitatively analyzed the complexion of patients, which is used as a diagnostic element in clinical practice. It is hoped that the results will contribute to objective medical treatments in the future, such as determining an appropriate herbal prescription based on the patient's complexion.
\end{abstract}

\section{Introduction}

In traditional Korean medicine (TKM), facial observation is one of the four examinations, and it is a factor in disease diagnosis [1]. In "The Treasure of Oriental Medicine" [2], numerous phrases are used to diagnose diseases and prescribe treatment according to patient's complexions, such as yellowish complexion, blue complexion, dark and blue complexion, white and dirty complexion, silver complexion, and red and shiny complexion. Furthermore, it is known that the complexions of each facial area reflect the state of the five viscera. It is also believed that the diseases of these five viscera and the complexions are related to each other.

The groups of disease in TKM can be divided into Cold and Heat patterns. Facial color, in addition to Cold and Heat, thirst, bowel and bladder function, tongue coat, and pulse, is an important differentiating factor in distinguishing these two patterns [1]. Moreover, in "The Yellow Emperor's Canon
Internal Medicine" [3], it is stated that the complexion and Cold-Heat pattern are closely related ("when a saint looks at a person's complexion, the yellow and red color sees it as having a lot of heat, while the blue and white color means that it has less heat, and the black color has a lot of blood and a little energy").

In Lee's book, "Longevity and Life Preservation in Oriental Medicine" [4], it is stated that the severity and the pattern changing of diseases are determined through the complexion. In the case of the So-Eum type, a blue complexion primarily appears when the conditions of the illness are severe or worsened, and yellow and red are expressed as a complexion when the illness is not severe or is subsiding. Lee claimed that the complexion reflects both the Sasang constitution and the state of the body.

In modern times, various elements related to the complexion have been studied. One of the elements is blood pressure. Skin color has been associated with both the systolic 
and diastolic blood pressure [5]. Specifically, a dark skin color is known to be associated with high blood pressure [6]. Additionally, with aging, the complexion tends to become dark, red, and yellow [7]. In medical studies, a difference in the complexion has been observed according to the degree of health; compared to the healthy group, the disease group was darker, and the subhealthy group was more pale [8]. Another study also examined the relationship between diseases and the complexion and showed that, in kidney disease, the complexion appeared dark, whereas in the case of hypertension or heart failure, a dark red color appeared [9].

While the relevance of the complexion to diseases in Western medicine is a subject that has been studied recently, the complexion is an essential diagnostic factor in TKM for diagnosing a disease and determining the prescription. Attempts have been made to quantitatively analyze the complexion to use this important factor objectively in TKM diagnoses [10]. One study examined the use of the complexion as a diagnostic element in SCM, in addition to body shape or symptoms [11, 12]. Although some studies have examined the use of the complexion as a diagnostic factor, objective evidence based on clinical data has been insufficient. In this study, we used objective evidence based on actual clinical data so that the complexion could be used in the objective diagnosis and treatment of SCM.

\section{Materials and Methods}

2.1. Data and Classification. Sasang medicine prescriptions collected by the Korea Constitutional Multicenter Bank (KCMB) were analyzed in this study. The data cases were taken, primarily the symptoms and progress of those patients that were given Sasang herbal medicine by doctors in Korean medicine clinics from 2013 to 2015. Diagnosis of Sasang type was performed by an expert using Sasang Constitution Analysis Tool (SCAT) [13]. Among the 915 subjects, data on 551 Tae-Eum-type, So-Eum-type, and So-Yang-type patients who were prescribed Taeumjowi-tang, Choweseunchengtang, Galgeunhaegi-tang, Yuldahanso-tang, Bojungikgitang, Jeokbaekhaogwanjung-tang, Gwankeibujalijung-tang, Baekhaolijung-tang, Hyangsayangwi-tang, Palmulgunja-tang, Hyungbangsabaek-san, Hyeongbangdojeok-san, Hyeongbangjihwang-tang, and Yanggyeoksanhwa-tang were selected. As 23 cases of complexion data failed to be extracted from the image, analysis was performed using data from 528 cases. Tae-Yang-type patients were excluded due to lack of subjects. Each Sasang prescription was classified into either a Cold- or Heat-prescription group (Table 1). Classification of Cold- and Heat-prescription group was based on "Longevity and Life Preservation in Oriental Medicine" [4].

2.2. General Characteristics of Subjects. Some significant differences were observed in the general characteristics of female (Table 2). For the So-Eum type, age of the Coldprescription group was higher than that of the Heatprescription group ( $p=0.008)$, whereas weight of the Heat-prescription group was higher than that of the Coldprescription group $(p=0.005)$. For the So-Yang type, age

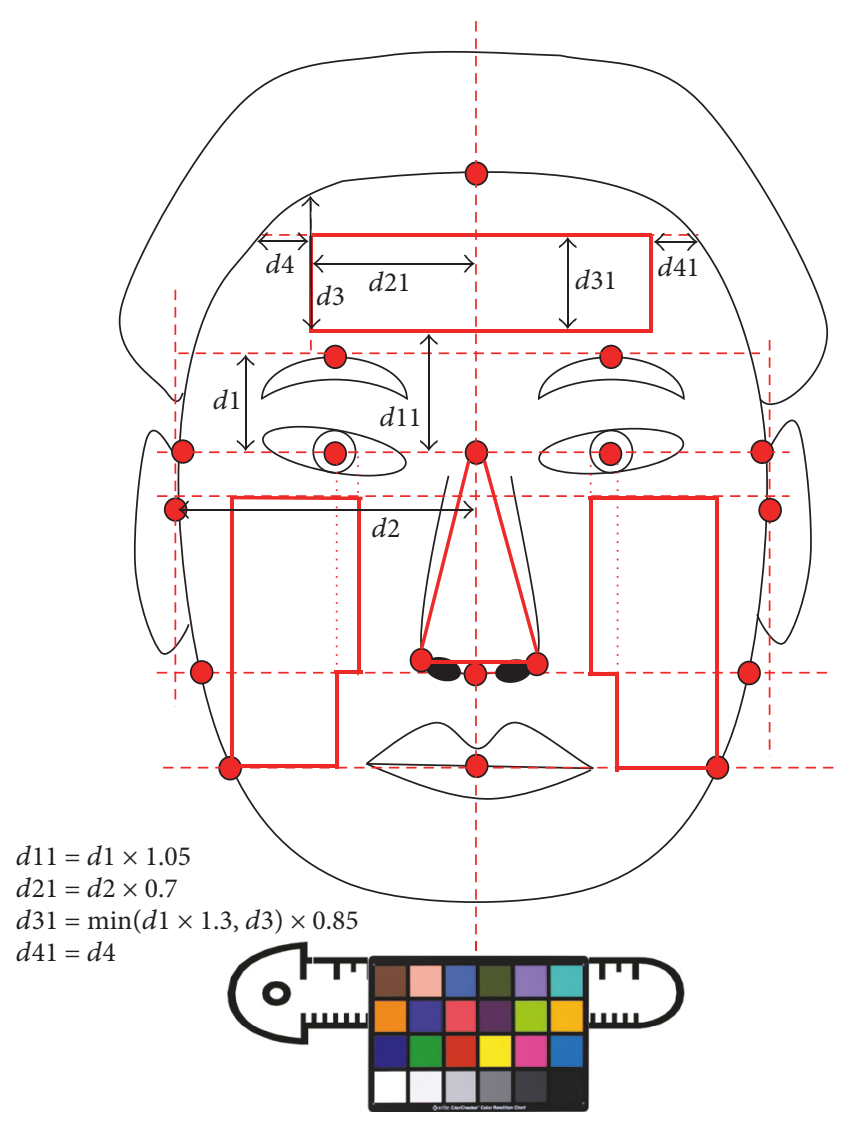

FIGURE 1: Facial complexion regions for the forehead, cheek, and nose. The regions are defined by the facial landmarks.

of Heat-prescription group was higher than that of the Coldprescription group $(p=0.002)$. But no significant differences were observed in other characteristics or in males. BMI and age were adjusted in the analysis.

\subsection{Photo Processing}

2.3.1. Facial Photography. A frontal face photograph was obtained according to a Standard Operation Process (SOP). Then, a color chart was attached to a reference ruler for color correction and placed approximately $1 \mathrm{~cm}$ below the subject's chin (Figure 1) [14].

\subsubsection{Color Correction Process}

(1) The position of the color chart was automatically recognized in the image, and the color information of each cell in the color chart was extracted.

(2) A color conversion model was generated by analyzing the relationship between the extracted color information and the reference color information of the color chart.

(3) The generated conversion model was applied to the input image to convert the color of the input image. 


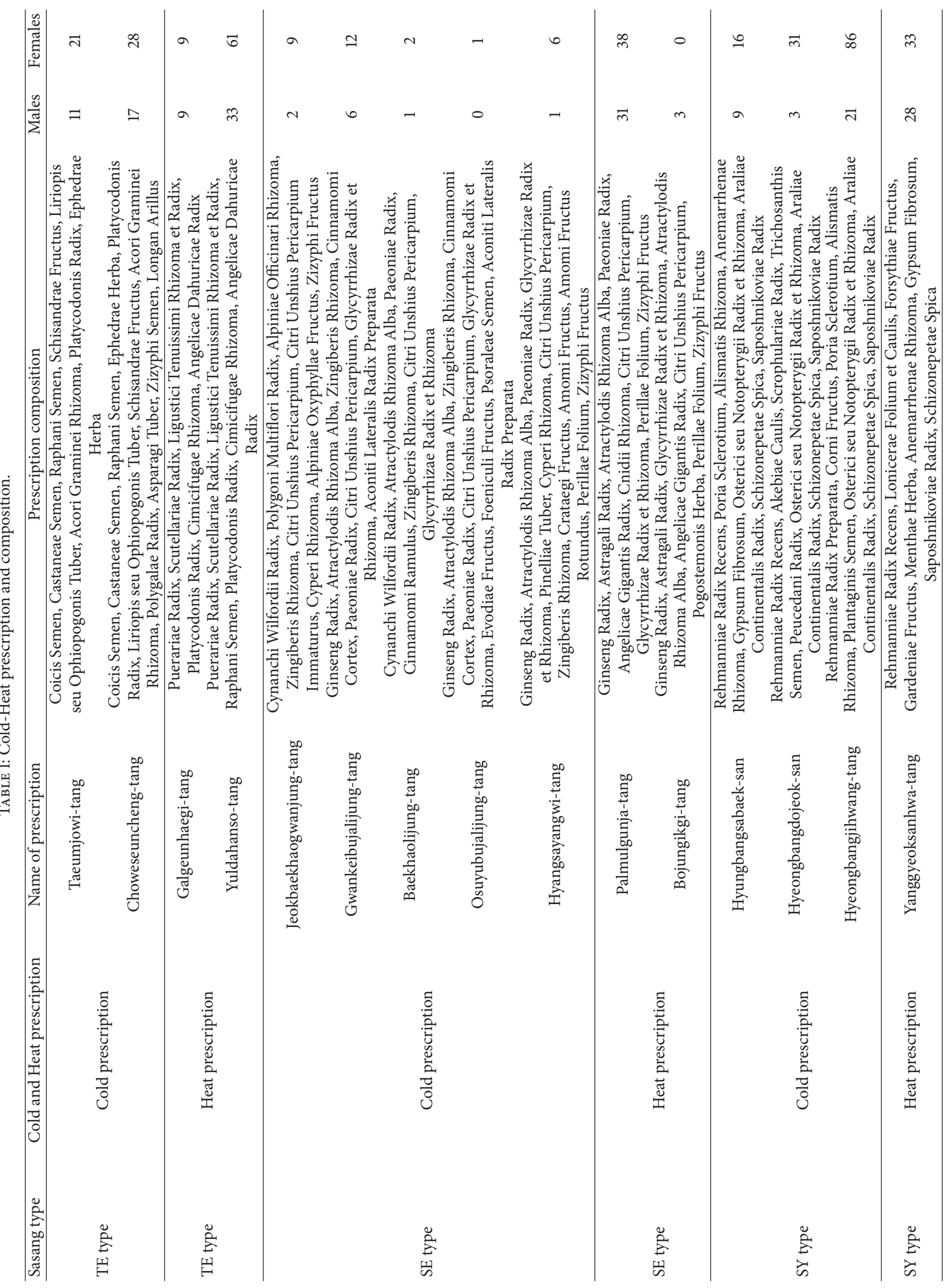


TABLE 2: General characteristics of the subjects.

\begin{tabular}{|c|c|c|c|c|c|c|c|c|c|}
\hline & \multicolumn{3}{|c|}{ TE type } & \multicolumn{3}{|c|}{ SE type } & \multicolumn{3}{|c|}{ SY type } \\
\hline & CG & HG & $p$ value & CG & HG & $p$ value & CG & HG & $p$ value \\
\hline \multicolumn{10}{|l|}{ Males } \\
\hline$N$ & 28 & 42 & & 10 & 34 & & 33 & 28 & \\
\hline Age & $45.11 \pm 2.79$ & $47.74 \pm 2.55$ & 0.499 & $43.38 \pm 4.39$ & $43.10 \pm 2.78$ & 0.962 & $43.32 \pm 2.40$ & $49.97 \pm 2.86$ & 0.078 \\
\hline Height & $172.37 \pm 1.15$ & $171.57 \pm 1.08$ & 0.622 & $168.93 \pm 2.03$ & $171.1 \pm 0.98$ & 0.310 & $172.49 \pm 1.13$ & $171.03 \pm 1.26$ & 0.390 \\
\hline Weight & $77.76 \pm 1.70$ & $76.26 \pm 1.52$ & 0.520 & $60.86 \pm 1.64$ & $63.70 \pm 1.40$ & 0.200 & $70.62 \pm 1.43$ & $70.72 \pm 1.69$ & 0.962 \\
\hline BMI & $26.24 \pm 0.63$ & $25.86 \pm 0.40$ & 0.594 & $21.41 \pm 0.79$ & $21.76 \pm 0.44$ & 0.706 & $23.73 \pm 0.41$ & $24.16 \pm 0.50$ & 0.505 \\
\hline \multicolumn{10}{|l|}{ Females } \\
\hline$N$ & 49 & 70 & & 30 & 38 & & 133 & 33 & \\
\hline Age & $44.78 \pm 2.04$ & $47.09 \pm 1.78$ & 0.399 & $49.55 \pm 2.82$ & $39.34 \pm 2.44$ & $0.008^{* *}$ & $44.28 \pm 1.27$ & $53.20 \pm 2.66$ & $0.002^{* *}$ \\
\hline Height & $160.08 \pm 0.76$ & $159.75 \pm 0.61$ & 0.734 & $157.31 \pm 1.10$ & $159.71 \pm 0.96$ & 0.105 & $158.23 \pm 0.50$ & $157.02 \pm 0.93$ & 0.274 \\
\hline Weight & $64.10 \pm 0.99$ & $65.26 \pm 0.98$ & 0.422 & $49.58 \pm 1.10$ & $53.91 \pm 1.01$ & $0.005^{* *}$ & $54.60 \pm 0.64$ & $55.33 \pm 1.14$ & 0.604 \\
\hline BMI & $25.04 \pm 0.38$ & $25.60 \pm 0.39$ & 0.319 & $20.07 \pm 0.44$ & $21.15 \pm 0.37$ & 0.061 & $21.81 \pm 0.25$ & $22.44 \pm 0.43$ & 0.251 \\
\hline
\end{tabular}

Data are presented in means \pm standard error. CG, Cold-prescription group; HG, Heat-prescription group. ${ }^{* *} p<0.01$. The analysis of statistical data was performed using $t$-test.

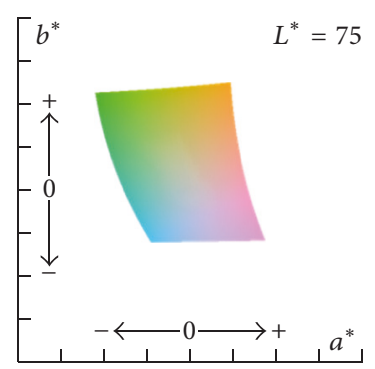

(a)

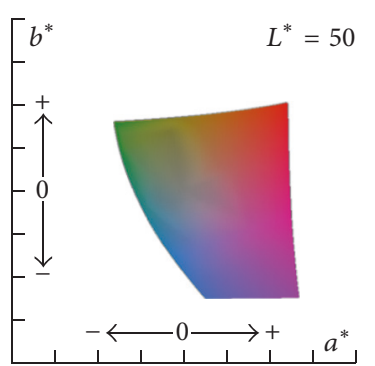

(b)

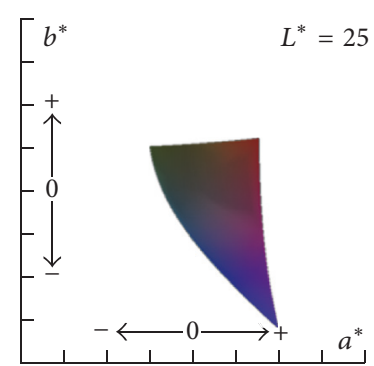

(c)

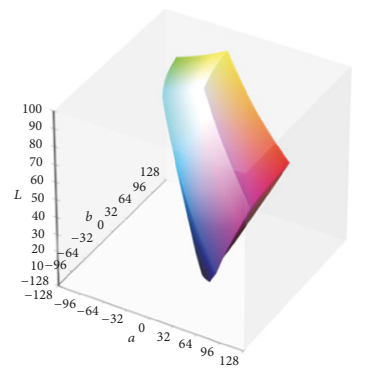

(d)

FIGURe 2: $L^{*} a^{*} b^{*}$ color space [15]. (a) sRGB gamut when $L^{*}$ value is 75. (b) sRGB gamut when $L^{*}$ value is 50. (c) sRGB gamut when $L^{*}$ value is 25. (d) sRGB gamut within $L^{*} a^{*} b^{*}$ color space.

\subsubsection{Detection of the Complexion Regions}

(1) Facial landmarks used to define the complexion regions were detected automatically using an image processing technique (Figure 1).

(2) Three complexion regions (forehead, cheek, and nose) were defined using the detected facial landmarks. And total is the average $L^{*} a^{*} b^{*}$ value calculated using the $L^{*} a^{*} b^{*}$ value of three regions (forehead, cheek, and nose).

\subsubsection{Extraction of Three Variables in the Complexion Regions}

(1) The red (R), green (G), and blue (B) values of each pixel in the complexion region were extracted.

(2) Because the $R, G$, and $B$ values are sensitive to illumination changes, these values were converted into $L^{*}$ values for lightness and $a^{*}$ and $b^{*}$ values for color-opponent dimensions (Figure 2) [15].

(3) The mean value of $L^{*}, a^{*}$, and $b^{*}$ in each pixel in the complexion region was calculated.
2.4. Statistical Analysis. The relationships of the complexion with age, BMI, and systolic blood pressure were analyzed using Pearson's correlation coefficient. The $L^{*} a^{*} b^{*}$ components of each facial region were analyzed using analysis of covariance (ANCOVA), and the age, BMI, and systolic blood pressure were used as covariates. SPSS 20 software (IBM Corp., Armonk, NY, USA) was used for the statistical analyses.

2.5. Institutional Review Board (IRB). This study was approved by the IRB of the Korea Institute of Oriental Medicine (I-1210/002-002-03).

\section{Results}

3.1. The Relationships of the Complexion with the Age, BMI, and Systolic Blood Pressure. Figure 3 shows the relationship between the value of $L^{*} a^{*} b^{*}$ in complexion and the age. In males, the age and $L^{*}$ values showed a negative correlation, indicating that the complexion darkens as the age increases. The age and both $a^{*}$ and $b^{*}$ values were positively correlated in both sexes, which indicates that, with increasing age, the complexion becomes more red and yellow. 


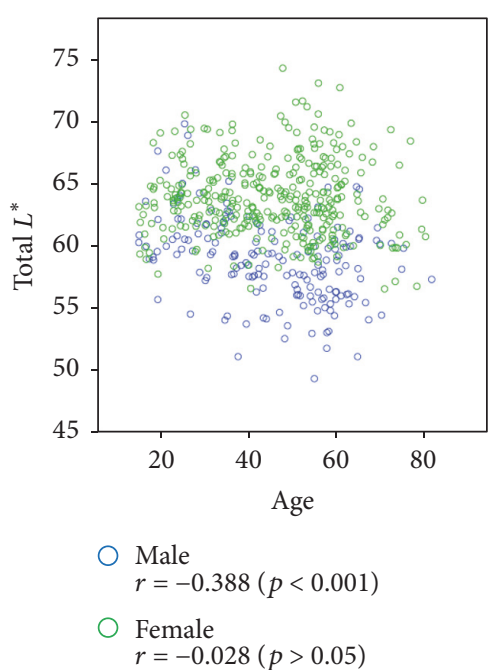

(a)

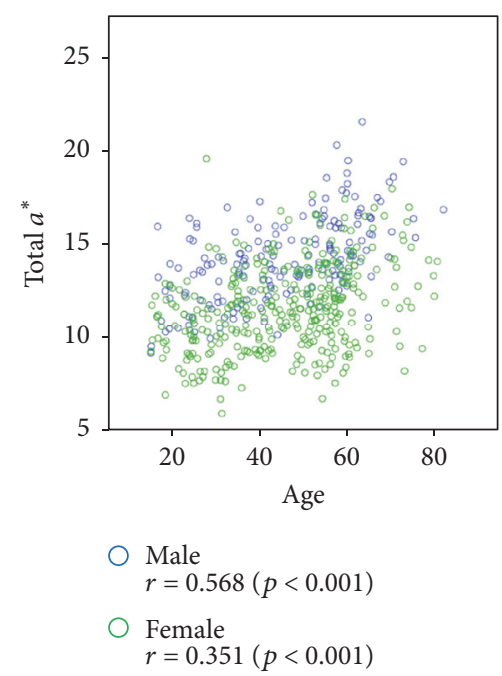

(b)

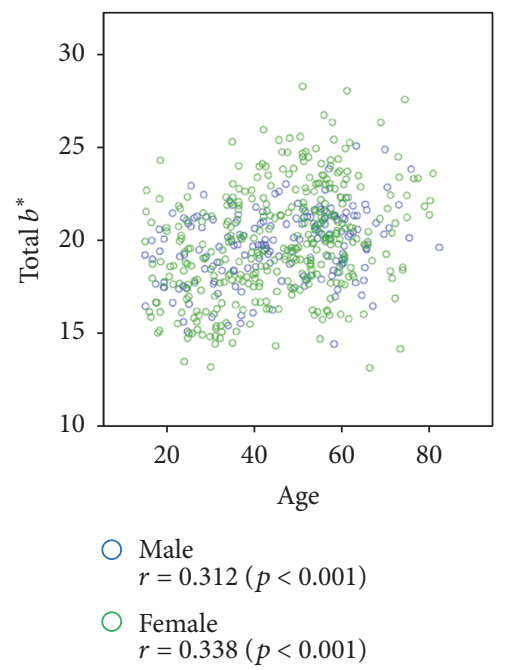

(c)

Figure 3: Scatter plot of the $L^{*} a^{*} b^{*}$ values versus age. Pearson correlation coefficients for associations of total $L^{*}$, total $a^{*}$, and total $b^{*}$ values with age are presented in the figures with $p$ value. (a) Scatter plot of the total $L^{*}$ value versus age. (b) Scatter plot of the total $a^{*}$ value versus age. (c) Scatter plot of the total $b^{*}$ value versus age.

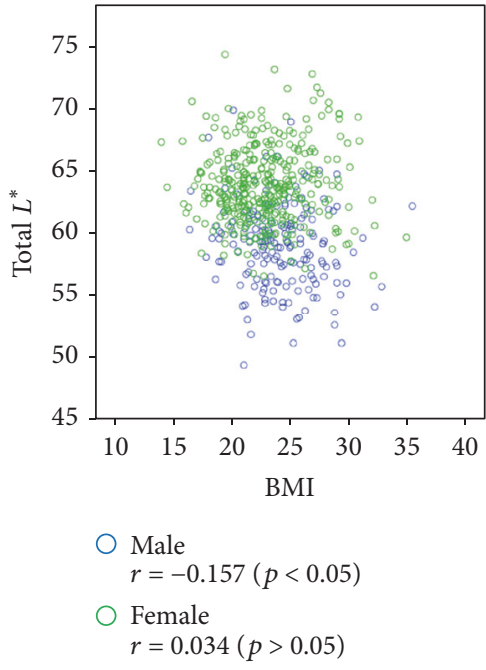

(a)

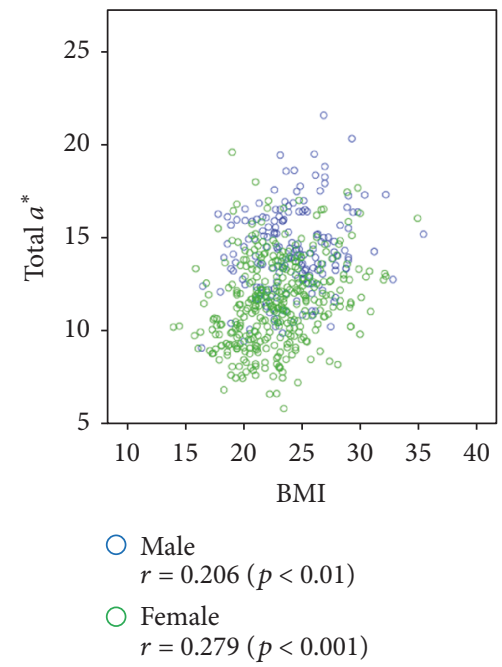

(b)

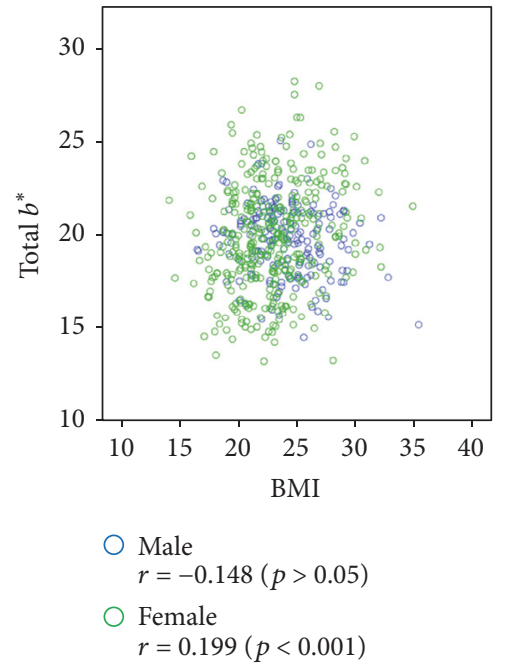

(c)

Figure 4: Scatter plot of the $L^{*} a^{*} b^{*}$ values versus BMI. Pearson correlation coefficients for associations of total $L^{*}$, total $a^{*}$, and total $b^{*}$ values with BMI are presented in the figures with $p$ value. (a) Scatter plot of the total $L^{*}$ value versus BMI. (b) Scatter plot of the total $a^{*}$ value versus BMI. (c) Scatter plot of the total $b^{*}$ value versus BMI.

Figure 4 shows the relationship between the value of $L^{*} a^{*} b^{*}$ in complexion and the BMI. In males, the BMI and $L^{*}$ values showed a negative correlation, indicating that as the BMI increases, the complexion becomes darker. In both men and women, a positive correlation was observed between the BMI and $a^{*}$ value, indicating that as the BMI increases, the complexion tends to become more complex. In addition, a positive correlation was found between the BMI and $b^{*}$ values in females, indicating that as the BMI increases, the complexion becomes increasingly associated with yellow.

Finally, we examined the relationship between the systolic blood pressure and the value of $L^{*} a^{*} b^{*}$ in complexion in
Figure 5. The systolic blood pressure and $a^{*}$ values were positively correlated in both males and females; thus, as the systolic blood pressure increased, the complexion became redder.

3.2. Analysis of Facial Complexion according to Cold- and Heat-Prescription Groups in Each Sasang Type. Table 3 shows the facial complexion analysis for males. For the Tae-Eum type, the $L^{*}$ value of the cheek and the $a^{*}$ value of the whole face, forehead, and nose of the Heat-prescription group were larger than those of the Cold-prescription group ( $p=0.018$, $0.045,0.016$, and 0.019 , resp.). For the So-Eum type, the $b^{*}$ 
TABLE 3: Facial complexion for males according to Cold- and Heat-prescription group.

\begin{tabular}{|c|c|c|c|c|c|c|c|c|c|c|}
\hline \multirow{2}{*}{ Region } & \multirow{2}{*}{$L^{*} a^{*} b^{*}$ color } & \multirow{2}{*}{ Group } & \multicolumn{2}{|c|}{ All males } & \multicolumn{2}{|c|}{ Tae-Eum type } & \multicolumn{2}{|c|}{ So-Eum type } & \multicolumn{2}{|c|}{ So-Yang type } \\
\hline & & & Mean \pm SE & $p$ value & Mean \pm SE & $p$ value & Mean \pm SE & $p$ value & Mean \pm SE & $p$ value \\
\hline \multirow{6}{*}{ Total } & \multirow{2}{*}{$L^{*}$} & CG & $58.99 \pm 0.37$ & \multirow{2}{*}{0.666} & $58.29 \pm 0.53$ & \multirow{2}{*}{0.151} & $59.84 \pm 1.13$ & \multirow{2}{*}{0.942} & $58.95 \pm 0.53$ & \multirow{2}{*}{$0.023^{*}$} \\
\hline & & HG & $58.78 \pm 0.31$ & & $59.28 \pm 0.43$ & & $59.94 \pm 0.61$ & & $57.08 \pm 0.58$ & \\
\hline & \multirow{2}{*}{$a^{*}$} & CG & $13.98 \pm 0.21$ & \multirow{2}{*}{0.192} & $14.18 \pm 0.33$ & \multirow{2}{*}{$0.045^{*}$} & $12.65 \pm 0.52$ & \multirow{2}{*}{0.128} & $14.40 \pm 0.27$ & \multirow{2}{*}{0.264} \\
\hline & & HG & $14.34 \pm 0.18$ & & $15.06 \pm 0.27$ & & $13.57 \pm 0.28$ & & $13.93 \pm 0.30$ & \\
\hline & \multirow{2}{*}{$b^{*}$} & CG & $19.62 \pm 0.21$ & \multirow{2}{*}{0.838} & $19.20 \pm 0.32$ & \multirow{2}{*}{0.307} & $19.00 \pm 0.52$ & \multirow{2}{*}{$0.049^{*}$} & $20.09 \pm 0.33$ & \multirow{2}{*}{0.072} \\
\hline & & HG & $19.68 \pm 0.18$ & & $19.63 \pm 0.26$ & & $20.21 \pm 0.28$ & & $19.17 \pm 0.36$ & \\
\hline \multirow{6}{*}{ Forehead } & \multirow{2}{*}{$L^{*}$} & CG & $63.52 \pm 0.47$ & \multirow{2}{*}{0.959} & $62.91 \pm 0.72$ & \multirow{2}{*}{0.694} & $63.66 \pm 1.46$ & \multirow{2}{*}{0.191} & $63.66 \pm 0.61$ & \multirow{2}{*}{$0.013^{*}$} \\
\hline & & HG & $63.49 \pm 0.40$ & & $63.28 \pm 0.59$ & & $65.88 \pm 0.79$ & & $61.31 \pm 0.66$ & \\
\hline & \multirow{2}{*}{$a^{*}$} & CG & $13.18 \pm 0.26$ & \multirow{2}{*}{0.209} & $13.22 \pm 0.04$ & & $11.73 \pm 0.67$ & 0.188 & $13.82 \pm 0.34$ & \\
\hline & & HG & $13.62 \pm 0.22$ & & $14.51 \pm 0.33$ & & $12.75 \pm 0.36$ & 0.188 & $13.00 \pm 0.37$ & \\
\hline & $b^{*}$ & CG & $20.66 \pm 0.26$ & 0.858 & $20.20 \pm 0.40$ & 0.680 & $19.86 \pm 0.63$ & $0.018^{*}$ & $21.33 \pm 0.37$ & $0.004^{* *}$ \\
\hline & & HG & $20.60 \pm 0.22$ & & $20.41 \pm 0.32$ & & $21.62 \pm 0.34$ & & $19.64 \pm 0.41$ & \\
\hline & $I^{*}$ & CG & $55.69 \pm 0.36$ & 0.515 & $55.03 \pm 0.48$ & $0.018^{*}$ & $56.42 \pm 1.12$ & 0.433 & $55.67 \pm 0.56$ & 0.067 \\
\hline & & HG & $55.38 \pm 0.31$ & ( & $56.53 \pm 0.39$ & & $55.41 \pm 0.60$ & & $54.08 \pm 0.61$ & \\
\hline Cheek & $a^{*}$ & CG & $14.22 \pm 0.20$ & 0233 & $14.57 \pm 0.32$ & 0166 & $13.10 \pm 0.50$ & 0205 & $14.41 \pm 0.27$ & 0730 \\
\hline & & HG & $14.54 \pm 0.17$ & 0.235 & $15.15 \pm 0.26$ & 0.100 & $13.84 \pm 0.27$ & 0.203 & $14.27 \pm 0.30$ & 0.730 \\
\hline & $b^{*}$ & CG & $18.98 \pm 0.22$ & 0.549 & $18.65 \pm 0.32$ & 0182 & $18.53 \pm 0.54$ & & $19.27 \pm 0.37$ & \\
\hline & $b$ & HG & $19.15 \pm 0.18$ & 0.549 & $19.20 \pm 0.26$ & 0.182 & $19.32 \pm 0.29$ & 0.201 & $18.99 \pm 0.40$ & $0.61 /$ \\
\hline & $L^{*}$ & CG & $63.14 \pm 0.45$ & & $62.31 \pm 0.71$ & & $64.97 \pm 1.36$ & 0.7 & $62.96 \pm 0.56$ & \\
\hline & & $\mathrm{HG}$ & $62.79 \pm 0.38$ & 0.558 & $62.01 \pm 0.58$ & 0.750 & $65.54 \pm 0.73$ & & $61.05 \pm 0.61$ & 0.028 \\
\hline Nose & $a^{*}$ & CG & $15.60 \pm 0.28$ & 0177 & $15.68 \pm 0.46$ & $0.019^{*}$ & $13.68 \pm 0.70$ & 0.065 & $16.38 \pm 0.36$ & 0.063 \\
\hline & & HG & $16.11 \pm 0.24$ & & $17.13 \pm 0.38$ & & $15.19 \pm 0.38$ & & $15.35 \pm 0.39$ & \\
\hline & $b^{*}$ & CG & $19.53 \pm 0.25$ & 0.488 & $18.85 \pm 0.36$ & 0.406 & $18.39 \pm 0.74$ & 0.051 & $20.36 \pm 0.35$ & $0.001^{* *}$ \\
\hline & & HG & $19.30 \pm 0.21$ & & $19.24 \pm 0.29$ & & $20.09 \pm 0.40$ & & $18.54 \pm 0.38$ & \\
\hline
\end{tabular}

Total is the average $L^{*} a^{*} b^{*}$ value calculated using the $L^{*} a^{*} b^{*}$ value of three regions (forehead, cheek, and nose). SE, standard error; CG, Cold-prescription group; HG, Heat-prescription group. ${ }^{*} p<0.05 ;{ }^{* *} p<0.01$. The analysis of statistical data was performed using ANCOVA. The mean and $p$ value were adjusted by covariates (age, BMI, and systolic blood pressure).

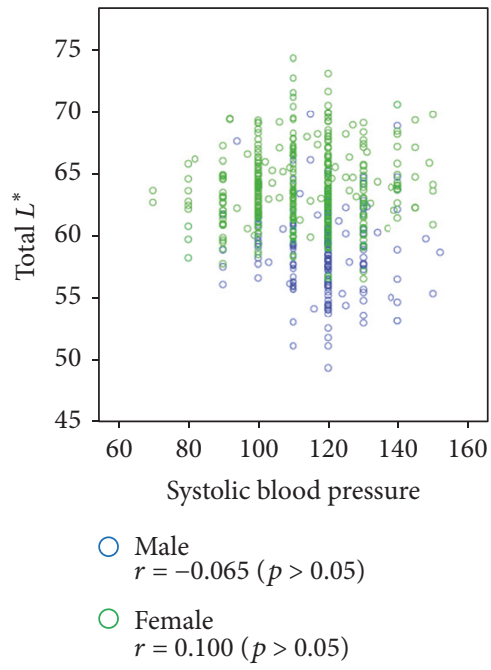

(a)

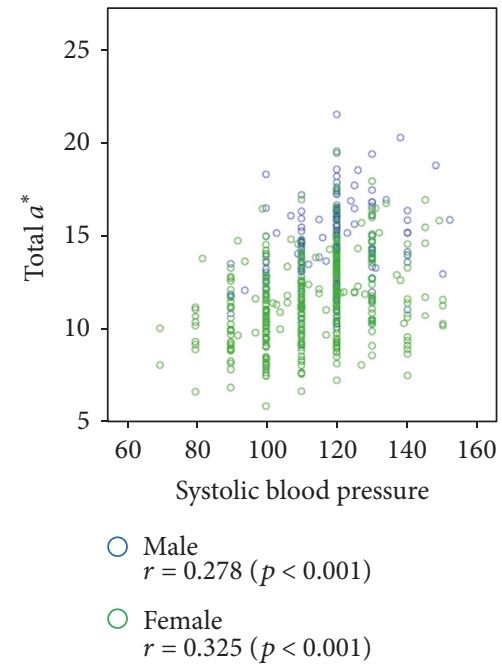

(b)

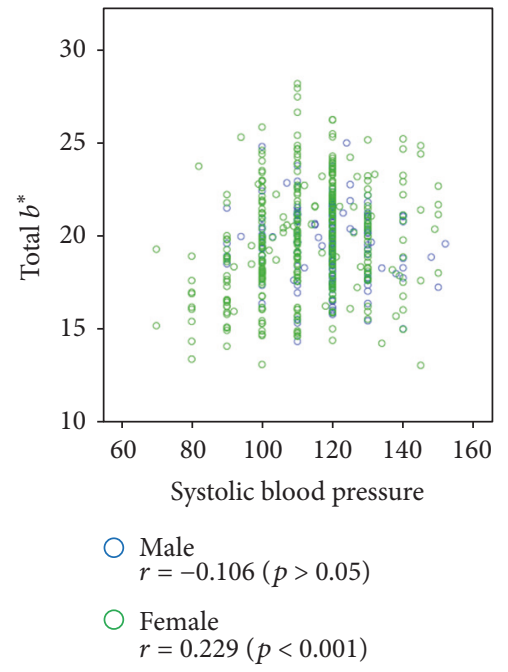

(c)

FIGURE 5: Scatter plot of the $L^{*} a^{*} b^{*}$ values versus systolic blood pressure (SBP). Pearson correlation coefficients for associations of total $L^{*}$, total $a^{*}$, and total $b^{*}$ values with SBP are presented in the figures with $p$ value. (a) Scatter plot of the total $L^{*}$ value versus SBP. (b) Scatter plot of the total $a^{*}$ value versus SBP. (c) Scatter plot of the total $b^{*}$ value versus SBP. 
TABLE 4: Facial complexion for females according to Cold- and Heat-prescription groups.

\begin{tabular}{|c|c|c|c|c|c|c|c|c|c|c|}
\hline \multirow{2}{*}{ Region } & \multirow{2}{*}{$L^{*} a^{*} b^{*}$ color } & \multirow{2}{*}{ Group } & \multicolumn{2}{|c|}{ All females } & \multicolumn{2}{|c|}{ Tae-Eum type } & \multicolumn{2}{|c|}{ So-Eum type } & \multicolumn{2}{|c|}{ So-Yang type } \\
\hline & & & Mean \pm SE & $p$ value & Mean \pm SE & $p$ value & Mean \pm SE & $p$ value & Mean \pm SE & $p$ value \\
\hline \multirow{6}{*}{ Total } & \multirow{2}{*}{$L^{*}$} & CG & $63.83 \pm 0.21$ & \multirow{2}{*}{0.848} & $63.83 \pm 0.49$ & \multirow{2}{*}{0.157} & $64.66 \pm 0.58$ & \multirow{2}{*}{0.137} & $63.65 \pm 0.23$ & \multirow{2}{*}{$0.041^{*}$} \\
\hline & & HG & $63.89 \pm 0.26$ & & $64.76 \pm 0.41$ & & $63.42 \pm 0.51$ & & $62.55 \pm 0.48$ & \\
\hline & \multirow{2}{*}{$a^{*}$} & CG & $11.50 \pm 0.15$ & \multirow{2}{*}{0.797} & $12.47 \pm 0.25$ & \multirow{2}{*}{0.096} & $11.01 \pm 0.38$ & \multirow{2}{*}{0.350} & $11.19 \pm 0.20$ & \multirow{2}{*}{0.207} \\
\hline & & HG & $11.44 \pm 0.18$ & & $11.92 \pm 0.21$ & & $10.51 \pm 0.33$ & & $11.79 \pm 0.42$ & \\
\hline & \multirow{2}{*}{$b^{*}$} & CG & $19.71 \pm 0.19$ & \multirow{2}{*}{0.513} & $20.34 \pm 0.36$ & \multirow{2}{*}{0.322} & $20.07 \pm 0.51$ & \multirow{2}{*}{0.321} & $19.36 \pm 0.24$ & \multirow{2}{*}{0.287} \\
\hline & & HG & $19.90 \pm 0.23$ & & $20.82 \pm 0.30$ & & $19.34 \pm 0.45$ & & $18.76 \pm 0.50$ & \\
\hline \multirow{6}{*}{ Forehead } & \multirow{2}{*}{$L^{*}$} & CG & $68.00 \pm 0.29$ & \multirow{2}{*}{0.109} & $67.92 \pm 0.68$ & \multirow{2}{*}{$0.006^{* *}$} & $68.99 \pm 0.78$ & \multirow{2}{*}{0.553} & $67.73 \pm 0.31$ & \multirow{2}{*}{$0.011^{*}$} \\
\hline & & HG & $68.74 \pm 0.35$ & & $70.42 \pm 0.57$ & & $68.33 \pm 0.68$ & & $65.93 \pm 0.62$ & \\
\hline & \multirow{2}{*}{$a^{*}$} & CG & $10.34 \pm 0.16$ & \multirow{2}{*}{0.585} & $11.12 \pm 0.29$ & 0.067 & $9.71 \pm 0.41$ & 0.740 & $10.13 \pm 0.22$ & 0.232 \\
\hline & & HG & $10.20 \pm 0.19$ & & $10.43 \pm 0.24$ & & $9.52 \pm 0.36$ & & $10.73 \pm 0.45$ & \\
\hline & $b^{*}$ & CG & $20.48 \pm 0.22$ & 0.416 & $21.30 \pm 0.43$ & 0.243 & $20.90 \pm 0.63$ & 0.345 & $20.00 \pm 0.27$ & 0.325 \\
\hline & & HG & $20.76 \pm 0.27$ & & $21.96 \pm 0.36$ & & $20.04 \pm 0.55$ & & $19.38 \pm 0.56$ & \\
\hline & $L^{*}$ & CG & $60.70 \pm 0.20$ & 0.176 & $61.01 \pm 0.46$ & 0.713 & $61.01 \pm 0.54$ & 0.051 & $60.56 \pm 0.25$ & 0.179 \\
\hline & & HG & $60.26 \pm 0.25$ & & $60.79 \pm 0.38$ & & $59.50 \pm 0.47$ & & $59.79 \pm 0.51$ & \\
\hline Cheek & $a^{*}$ & CG & $12.19 \pm 0.15$ & 0.924 & $13.19 \pm 0.26$ & 0.240 & $11.93 \pm 0.38$ & 0.168 & $11.82 \pm 0.20$ & $0.1 / 9$ \\
\hline & $a$ & HG & $12.22 \pm 0.18$ & 0.924 & $12.79 \pm 0.21$ & 0.240 & $11.18 \pm 0.33$ & & $12.44 \pm 0.41$ & \\
\hline & $b^{*}$ & CG & $19.33 \pm 0.18$ & 0.645 & $19.82 \pm 0.35$ & 0.395 & $19.63 \pm 0.48$ & 0.332 & $19.07 \pm 0.24$ & 0.264 \\
\hline & & HG & $19.46 \pm 0.22$ & & $20.21 \pm 0.29$ & & $18.97 \pm 0.42$ & & $18.46 \pm 0.49$ & \\
\hline & $L^{*}$ & CG & $67.71 \pm 0.30$ & 0.098 & $67.26 \pm 0.69$ & $0.006^{* *}$ & $69.91 \pm 0.86$ & 0.205 & $67.39 \pm 0.31$ & $0.042^{*}$ \\
\hline & & HG & $68.50 \pm 0.37$ & & $69.77 \pm 0.57$ & 0.000 & $68.36 \pm 0.75$ & 0.205 & $65.92 \pm 0.64$ & 0.042 \\
\hline Nose & $a^{*}$ & CG & $11.81 \pm 0.18$ & 0.423 & $13.05 \pm 0.33$ & $0.034^{*}$ & $10.77 \pm 0.46$ & 0.536 & $11.48 \pm 0.25$ & 0.163 \\
\hline & & HG & $11.58 \pm 0.22$ & & $12.12 \pm 0.28$ & & $10.36 \pm 0.41$ & & $12.29 \pm 0.51$ & \\
\hline & $b^{*}$ & CG & $19.04 \pm 0.20$ & 0354 & $19.96 \pm 0.39$ & 0.460 & $19.28 \pm 0.59$ & 0.505 & $18.57 \pm 0.24$ & 0.464 \\
\hline & 0 & HG & $19.33 \pm 0.24$ & 0.534 & $20.35 \pm 0.33$ & 0.400 & $18.72 \pm 0.52$ & & $18.17 \pm 0.50$ & \\
\hline
\end{tabular}

Total is the average $L^{*} a^{*} b^{*}$ value calculated using the $L^{*} a^{*} b^{*}$ value of three regions (forehead, cheek, and nose). SE, standard error; CG, Cold-prescription group; HG, Heat-prescription group. ${ }^{*} p<0.05 ;{ }^{* *} p<0.01$. The analysis of statistical data was performed using ANCOVA. The mean and $p$ value were adjusted by covariates (age, BMI, and systolic blood pressure).

value of the whole face and forehead of the Heat-prescription group was larger than that of the Cold-prescription group ( $p=0.049,0.018$, resp.). For the So-Yang type, the $L^{*}$ value of the whole face and forehead and the $b^{*}$ value of the forehead and nose of the Cold-prescription group were larger than those of the Heat-prescription group ( $p=0.023,0.013,0.004$, and 0.001 , resp.).

After the effects of the age, BMI, and systolic blood pressure on the complexion have been eliminated, significant differences were observed in the complexion according to the Cold- and Heat-prescription groups in each Sasang constitution. However, there was no significant difference in the male group as a whole.

Table 4 shows the facial complexion analysis for females. For the Tae-Eum type, the $L^{*}$ values of the forehead and nose of the Heat-prescription group were greater than those of the Cold-prescription group ( $p=0.006$ and 0.006 , resp.). In contrast to the males, the $a^{*}$ value of the nose in the Cold-prescription group was greater than that of the Heatprescription group ( $p=0.034)$. For the So-Yang type, the $L^{*}$ values of the whole face, forehead, and nose of the Cold-prescription group were greater than those of the Heatprescription group ( $p=0.041,0.011$, and 0.042 , resp.). However, there were no significant differences in the value of $L^{*} a^{*} b^{*}$ in complexion when Sasang constitution was not taken into consideration.

Although fewer differences were observed for females than for males, differences in the complexion were found according to the Cold- and Heat-prescription groups, despite the correction for the covariates of the age, BMI, and systolic blood pressure.

\section{Discussion}

Using the color space of $L^{*} a^{*} b^{*}$, we analyzed the differences in the complexion according to the Cold- and Heatprescription groups and obtained interesting results. No significant difference was observed in the complexion according to the Cold- and Heat-prescription groups for the overall male and female groups consisting of the Tae-Eum, So-Eum, and So-Yang types, but significant differences were found for each Sasang constitution. To date, differences according to Sasang constitution have been the primary focus of studies $[16,17]$, but our results show differences within the same Sasang constitution. In addition, the results were consistent with the TKM literature.

Firstly, in the "Longevity and Life Preservation in Oriental Medicine," for the Tae-Eum type, people with a blue and 
white facial color are stated to have fewer dryness syndromes, and people with a yellow, red, and black facial color have increased dryness syndromes. Thus, according to the literature, the Heat-pattern prescription group of the Tae-Eum type will often have a yellow, red, and black face, and the Cold-prescription group of the Tae-Eum type will have a blue or white face. In the analysis of this study, the $a^{*}$ value of the Heat-prescription group of the Tae-Eum type was significantly higher in males, and the complexion appeared red; however, this was not the case for females. Although not significant, the $b^{*}$ value of the Heat-prescription group of the Tae-Eum type was slightly higher for both sexes, and the color was yellowish.

The complexion may show the opposite tendency depending on the Sasang constitution. In the "Donguisusebowon Sasangchobonguan" [18], the complexion in healthy and sick individuals is different according to the Sasang constitution ("the So-Eum type is healthy when the complexion is soft violet, but ill when it is turbid yellow. In the case of the Tae-Eum type, it is healthy when the complexion is shiny violet. The So-Yang type is healthy when the complexion is shiny blue, but it is ill when it is white or black. In the case of the Tae-Yang type, it is healthy when the complexion is soft white but ill when the complexion is black"). In other words, the mechanism by which the physiological state is expressed as a facial color may vary depending on the Sasang constitution. In the analysis of this study, we also observed that the complexion of the Tae-Eum type and So-Yang type had opposite tendencies. The Tae-Eum type had a higher $L^{*}$ value in the Heat-prescription group and showed a bright complexion. In contrast, the So-Yang type had a higher $L^{*}$ value in the Cold-prescription group. Additionally, the $a^{*}$ and $b^{*}$ values differed by sex, but the two Sasang constitutions showed opposite tendencies.

According to the Sasang medical literature, the complexion is also different between the So-Eum and Tae-Eum types. In the "Donguisusebowon Sasangchobonguan" [18], when the face is oily, illness is thought to be worsened in the So-Eum type; however, this characteristic indicates improvement in illness in the Tae-Eum type. The analysis of this study confirmed that the complexions of the Tae-Eum and So-Eum types were not opposite but showed different tendencies. As described above, even in the case of the same Cold-prescription and Heat-prescription groups, the mechanism through which the complexion is expressed by the Sasang constitution is different; therefore, the analysis did not show any significant difference among the three Sasang constitutions.

In TKM, a person in the Cold-pattern group is characterized by sensitivity to cold and weak digestive power, whereas a person in the Heat-pattern group is characterized by sensitivity to heat and strong digestive power $[1,19]$. Even if the Sasang constitution is different, the Cold- and Heatpattern groups can be consistently classified according to clinical characteristics [20]. It is worthwhile to note that, unlike clinical symptoms, a significant difference was found in the analysis of the complexion only when the Sasang constitution was divided. It can be speculated that the factors related to Cold or Heat will affect the blood flow rate [21] and that various physiological functions will result in differences in the complexion. However, this study showed differences in the complexion related to the Sasang constitution as well as to the Cold-Heat pattern.

The Cold-Heat pattern is an important concept with high clinical value in TKM. The objectification and quantification of the complexion, a key diagnostic factor in determining the Cold-Heat pattern, are essential for the standardization of the diagnoses of TKM. This study showed a difference in the complexion among the Cold- and Heat-prescription groups by an objective method based on actual clinical data that will help determine the prescription. If larger scale clinical studies show the same results in the future, it will be possible to standardize diagnosis using the complexion. This will greatly contribute to enhancing the level of clinical medicine in TKM.

\section{Conclusions}

The complexion is an important diagnostic factor in Korean medicine and is related to the Sasang constitution. Based on actual clinical data, we found significant differences in the complexion between the Cold-prescription and Heatprescription groups, which showed different patterns according to the Sasang constitution. The results confirmed that when adjusted for the age, BMI, and systolic blood pressure, the Cold-Heat pattern in TKM is related to the complexion.

\section{Conflicts of Interest}

The authors declare no conflicts of interest.

\section{Acknowledgments}

This research was supported by the Bio \& Medical Technology Development Program of the National Research Foundation of Korea (NRF) funded by the Korean government and MSIP (NRF-2015M3A9B6027139). This research was also supported by the NRF funded by the Ministry of Science, ICT \& Future Planning (no. 2006-2005175).

\section{References}

[1] Teaching Material Compilation Committee of Oriental Pathology, Oriental Pathology, Hanuimunhwasa, Seoul, Republic of Korea, 2002.

[2] J. Heo, J. Jin, C. Kang et al., The Treasure of Oriental Medicine, Beopinmunhwasa, Seoul, Republic of Korea, 2012.

[3] D. Kim, Yellow Emperor's Canon Internal Medicine Ling Shu, Uiseongdang, Seoul, Republic of Korea, 2002.

[4] J. Lee and S. Choi, Longevity and Life Preservation in Oriental Medicine, Kyung Hee, Kyung Hee University Press, Seoul, Republic of Korea, 1966.

[5] C. C. Gravlee, W. W. Dressier, and H. Russell Bernard, "Skin color, social classification, and blood pressure in Southeastern Puerto Rico," American Journal of Public Health, vol. 95, no. 12, pp. 2191-2197, 2005.

[6] C. C. Gravlee and W. W. Dressler, "Skin pigmentation, selfperceived color, and arterial blood pressure in Puerto Rico," 
American Journal of Human Biology, vol. 17, no. 2, pp. 195-206, 2005.

[7] J. de Rigal, I. des Mazis, S. Diridollou et al., "The effect of age on skin color and color heterogeneity in four ethnic groups," Skin Research and Technology, vol. 16, no. 2, pp. 168-178, 2010.

[8] J. Xu, H. Wu, L. Lu, L. Tu, Z. Zhang, and X. Chen, "Analysis of different health status based on characteristics of the facial spectrum photometric color," Journal of Biomedical Engineering, vol. 29, no. 6, pp. 1062-1067, 2012.

[9] K. Kawano, M. Suzuki, and K. Araki, "Use of digital patient photographs and electronic medical record data as diagnostic tools in Japan," Journal of Medical Systems, vol. 36, no. 5, pp. 3321-3326, 2012.

[10] J. Do, B. Ku, J. Kim et al., "Quantitative analysis of face color according to health status of four constitution types for Korean elderly male," Journal of Korean Oriental Medicine, vol. 26, no. 1, pp. 128-132, 2012.

[11] J. U. Kim, B. Ku, Y.-M. Kim et al., "The concept of sasang health index and constitution-based health assessment: An integrative model with computerized four diagnosis methods," Evidence-based Complementary and Alternative Medicine, vol. 2013, Article ID 879420, 2013.

[12] Y. J. Park, J. Nam, J.-H. Do, H. J. Jin, and J. Y. Kim, "Bodily differences between cold- and heat-prescription groups in Sasang medicine," Integrative Medicine Research, vol. 5, no. 2, pp. 118-123, 2016.

[13] J.-H. Do, J.-H. Nam, E.-S. Jang et al., "Comparison between diagnostic results of the Sasang Constitutional Analysis Tool (SCAT) and a Sasang constitution expert," Journal of Sasang Constitutional Medicine, vol. 25, no. 3, pp. 158-166, 2013.

[14] S. Lee, Y. Kim, J. Seo, and J. Kim, Development of Diagnostic and Herbal Drug System Based on Traditional Constitutional Korean Medicine, Korea Institute of Oriental Medicine, Daejeon, Republic of Korea, 2007.

[15] Lab Color Space. Wikipedia. Available at: https://en.wikipedia .org/wiki/Lab_color_space. Accessed February 1, 2017.

[16] S. K. Lee, D. W. Yoon, H. Yi et al., "So-eum type as an independent risk factor for irritable bowel syndrome: a population-based study in Korea," The Journal of Alternative and Complementary Medicine, vol. 20, no. 11, pp. 846-852, 2014.

[17] S. K. Lee, D. W. Yoon, S. W. Lee et al., "Association of Sasang Constitutional Types with Incident Hypertension: A 12-Year Follow-Up Study," Journal of Alternative and Complementary Medicine, vol. 22, no. 9, pp. 706-712, 2016.

[18] J. Lee and S. Park, Donguisusebowon Sasangchobonguan. Jipmundang, Seoul, Republic of Korea, 2003.

[19] K.-H. Bae, J. A. Lee, K.-H. Park, J.-H. Yoo, Y. Lee, and S. Lee, "Cold Hypersensitivity in the Hands and Feet May Be Associated with Functional Dyspepsia: Results of a Multicenter Survey Study," Evidence-based Complementary and Alternative Medicine, vol. 2016, Article ID 8948690, 2016.

[20] H.-J. Jin, S.-H. Kim, S.-O. Dong, E.-S. Jang, and S.-W. Lee, “The agreement in cold-heat and health status among sasang constitutional experts in diagnosis of sasang pathological symptoms," Journal of Sasang Constitutional Medicine, vol. 26, no. 2, pp. 146155, 2014.

[21] N. Kakitsuba, "Physiological responses to changes in relative humidity under thermally neutral, warm and hot conditions," Journal of Thermal Biology, vol. 59, pp. 86-91, 2016. 


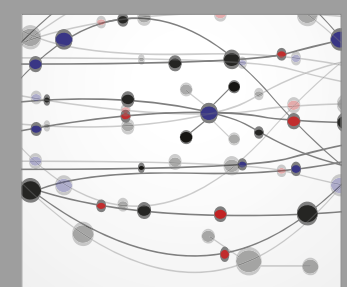

The Scientific World Journal
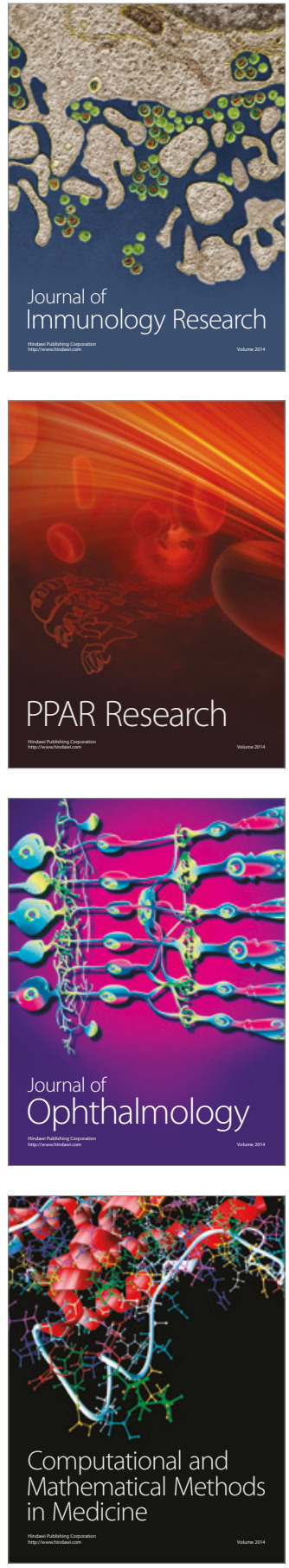

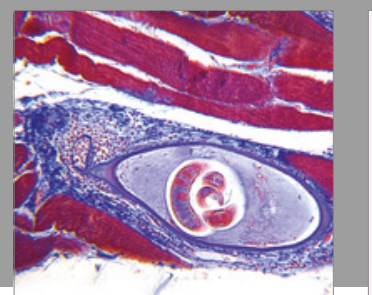

Gastroenterology Research and Practice
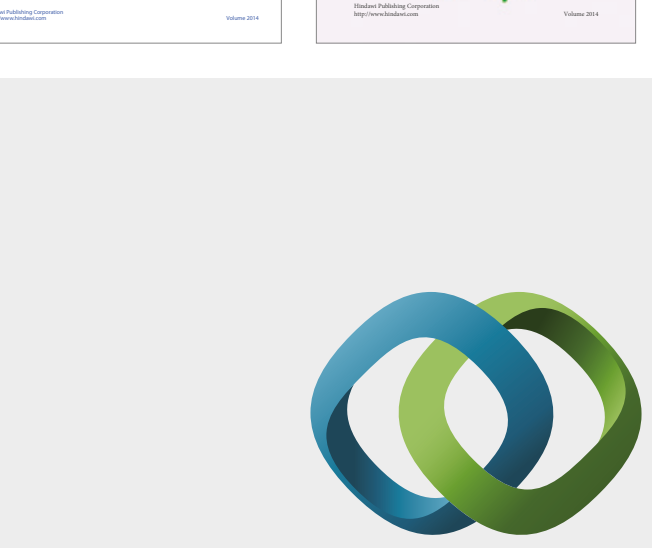

\section{Hindawi}

Submit your manuscripts at

https://www.hindawi.com
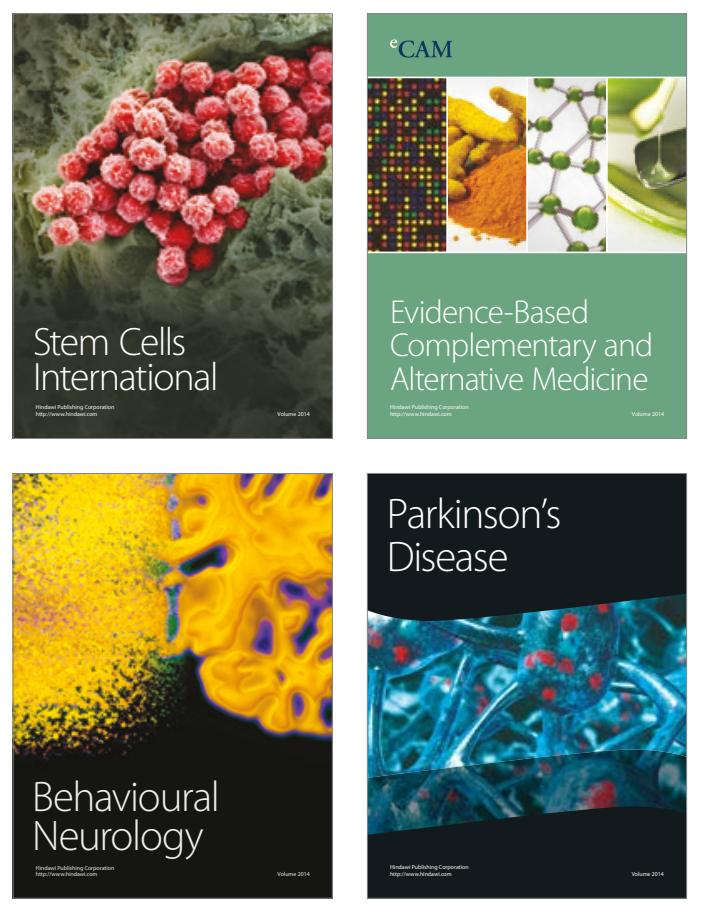
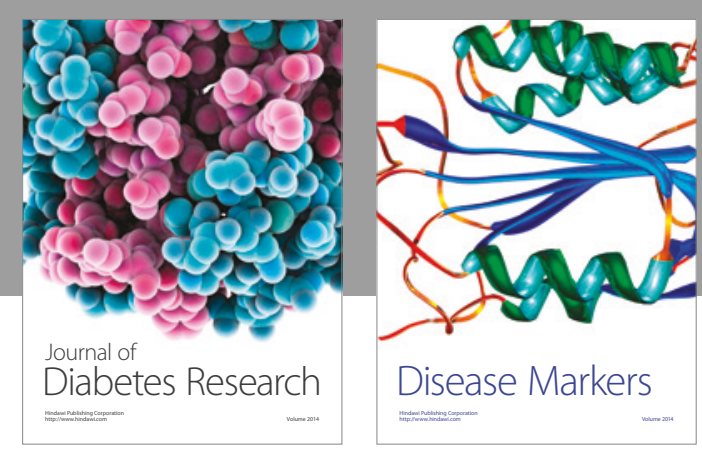

Disease Markers
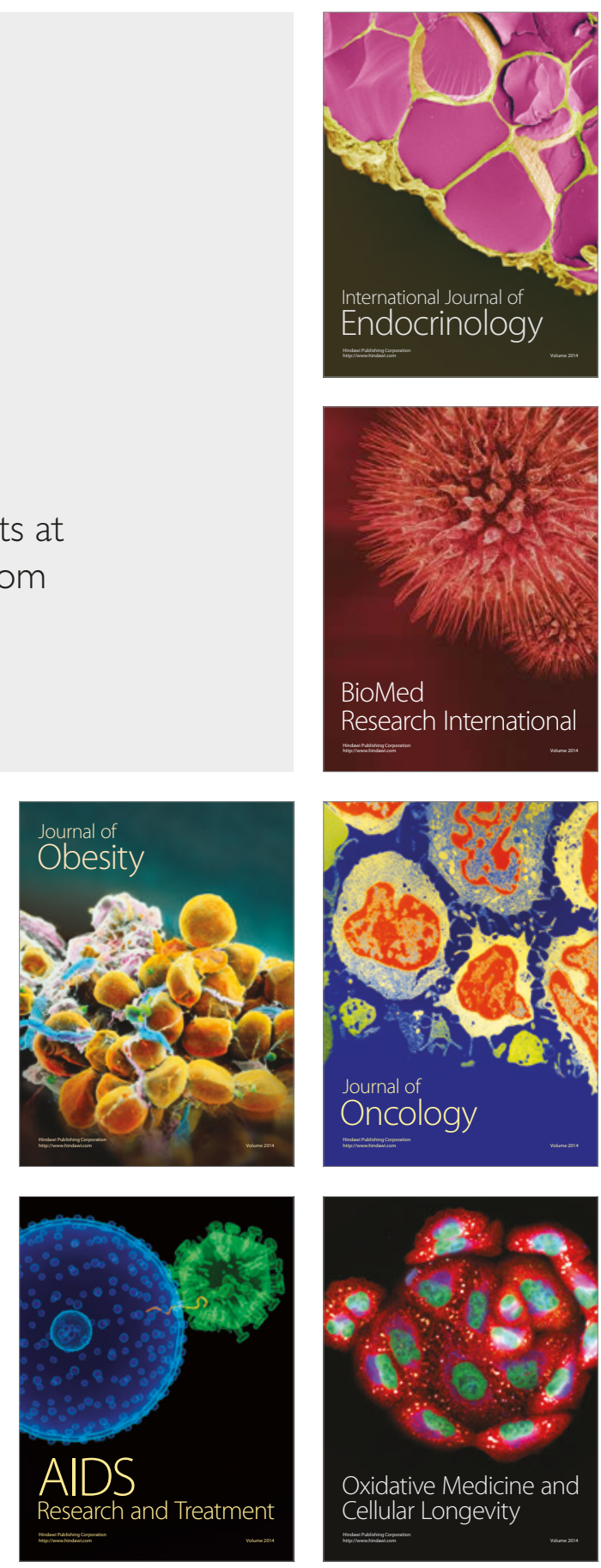